\title{
PERFIL DE ESTUDANTES ACOLHIDOS EM UM SERVIÇO DE SAÚDE NA UNIVERSIDADE
}

\section{Profile of students admitted to health service at university \\ Perfil de estudiantes de un servicio de salud de la universidad}

\author{
Luciola D'Emery Siqueira \\ Universidade Federal de São Paulo - UNIFESP - São Paulo (SP) - Brasil
}

Matheus Ferreira Guimarães Bastos

Universidade Federal de São Paulo - UNIFESP - São Paulo (SP) - Brasil

Andréia do Nascimento Santos

Universidade Federal de São Paulo - UNIFESP - São Paulo (SP) - Brasil

Mariana Puridade Marques da Silva

Universidade Federal de São Paulo - UNIFESP - São Paulo (SP) - Brasil

\section{RESUMO}

Objetivo: Caracterizar o perfil sociodemográfico e clínico de estudantes universitários acolhidos em um serviço de saúde. Métodos: Tratase de um estudo descritivo e retrospectivo, baseado na análise de prontuários de estudantes matriculados na Universidade Federal de São Paulo e que utilizaram os serviços de saúde entre os anos de 2011 e 2015. Foram utilizadas as variáveis: sexo, faixa etária, estado civil, curso, ocupação, tipo de moradia, consumo de tabaco, existência de doença pré-existente e motivo da procura pelo serviço. Resultados: Foram elegíveis 457 prontuários, sendo a maioria do sexo feminino $(n=318,69,6 \%)$, de 20 a 29 anos, residentes com seus familiares ( $n=304$; $66,5 \%$ ), cisgênero $(n=455 ; 99,5 \%)$ e atuantes no mercado de trabalho. Quanto ao motivo do acolhimento, apesar de $72 \%(n=330)$ não possuir doenças preexistentes, metade dos estudantes solicitaram encaminhamento para médico. Conclusão: O estudo evidenciou que a maioria dos estudantes que procuraram os serviços de saúde eram mulheres, na faixa etária de 20 a 29 anos, residentes com suas famílias e atuantes ao mercado de trabalho. Embora a maioria dos estudantes não referir doenças preexistente, havia relações dessas questões psicossociais, doenças crônicas e gastrointestinais.

Descritores: Serviços de Saúde para Estudantes; Perfil de Saúde; Promoção da Saúde.

\section{ABSTRACT}

Objective: To characterize the sociodemographic and clinical profiles of students admitted to a health service. Methods: Descriptive and retrospective study based on the analysis of medical records of students enrolled at the Federal University of São Paulo who used the health services between 2011 and 2015. The following variables were used to identify students'sociodemographic and clinical profiles: sex, gender, age, occupation, type of housing, tobacco consumption, preexisting disease and reason for using the service. Results: a total of 457 medical records were eligible; most participants were women $(n=318,69.6 \%)$, aged 20 to 29 years, lived with their families $(n=304,66.5 \%)$, were cisgender $(n=455,99.5 \%)$ and worked. The reasons for seeking the health service included the need to be referred to aa specialist, admission and assessment, and psychosocial complaints, although $72 \%(n=330)$ of the participants did not have preexisting diseases. Conclusion: The students admitted to the health service are mostly women, aged 20 to 29 years old, lived with their families and worked. Although most of the students did not present with preexisting diseases, their health complaints were related to psychosocial issues and chronic and gastrointestinal diseases.

Descriptors: Student Health Service; Health Profile; Health Promotion. 


\section{RESUMEN}

Objetivo: Caracterizar el perfil clínico y sociodemográfico de estudiantes universitarios de un servicio de salud. Métodos: Se trata de un estudio descriptivo y retrospectivo basado en el análisis de historiales clínicos de estudiantes matriculados en la Universidad Federal de São Paulo que han utilizado los servicios de salud entre 2011 y 2015. Para identificar el perfil clínico y sociodemográfico de los estudiantes se utilizaron las variables de sexo, género, franja de edad, ocupación, tipo de vivienda, consumo de tabaco, existencia de enfermedad preexistente y el motivo para la busca del servicio. Resultados: 457 historiales clínicos fueron elegibles siendo la mayoría del sexo femenino ( $n=318,69,6 \%)$ entre 20 y 29 años, que viven con sus familiares $(n=304,66,5 \%)$, cisgênero $(n=455,99,5 \%)$ y que actúan en el mercado de trabajo. El motivo de las consultas se dieron por la necesidad de la indicación para un especialista, la acogida y la evaluación, y quejas psicosociales a pesar del 72\% (n=330) no tener enfermedades preexistentes. Conclusión: En el perfil de los estudiantes asistidos en el servicio de salud predominan mujeres en la franja de edad entre 20 y 29 años, que viven con sus familias y actúan en el mercado de trabajo. A pesar de la mayoría no referir enfermedades preexistentes, habian relaciones de estas con aspectos psicosociales, enfermedades crónicas y gastrointestinales.

Descriptores: Servicios de Salud para Estudiantes; Perfil de Salud; Promoción de la Salud.

\section{INTRODUÇÃO}

Nas duas últimas décadas, ocorreram intensas modificações no ensino superior brasileiro. Especificamente no ensino público, priorizou-se a ampliação do acesso, com a diversificação das formas de ingresso e aumento do quantitativo das vagas no ensino superior, o que repercutiu intensamente no perfil do estudante universitário. O ensino superior, antes reduto de classes socioeconômicas mais favorecidas, torna-se aos poucos mais acessível para várias camadas da sociedade, o que tem modificado o perfil do estudante universitário brasileiro ${ }^{(1)}$.

Um levantamento sobre o perfil desse "novo" estudante evidenciou que 53,5\% são mulheres, $75 \%$ estão na faixa etária de 18 a 24 anos, $88,6 \%$ são solteiros e 54\% são de cor/raça branca, apesar do gradual aumento no percentual de pretos e pardos na universidade. Quanto ao local de moradia, 55,3\% residem com os pais/familiares e um terço exerce atividade remunerada ${ }^{(2)}$.

No entanto, apenas ampliar o acesso à universidade não é suficiente para garantir a permanência do estudante e sua efetiva conclusão do curso. Faz-se necessária a adoção de programas de assistência estudantil para estudantes em situação de vulnerabilidade socioeconômica, como o Programa Nacional de Assistência Estudantil - PNAES, que tem como objetivos: democratizar as condições de permanência dos jovens na educação superior pública federal; minimizar os efeitos das desigualdades sociais e regionais na permanência e conclusão do ensino superior; reduzir as taxas de retenção e evasão; e contribuir para a inclusão social pela educação ${ }^{(3)}$.

As condições de saúde e qualidade de vida do estudante estão incluídas como diretrizes do PNAES, sendo desenvolvidas ações nas áreas de moradia, alimentação, transporte, atenção à saúde, inclusão digital, cultura, esporte, creche, apoio pedagógico e inclusão do estudante com deficiência ${ }^{(3)}$.

O PNAES incorpora em suas ações a temática da promoção de saúde quando busca um paradigma de saúde voltado para o bem-estar e desenvolvimento sustentável, utilizando todas as oportunidades disponíveis de forma intersetorial ${ }^{(4)}$, fortalecendo as capacidades do estudante e transformando o ambiente acadêmico em favorável para a adoção de hábitos saudáveis pela comunidade estudantil.

O Programa de Apoio a Planos de Reestruturação e Expansão das Universidades Federais (REUNI) ampliou o acesso à universidade pública federal, sendo o PNAES uma ferramenta para a concretização dessa expansão, principalmente considerando a relevâncias das universidades públicas para a produção científica brasileira e visibilidade internacional do desenvolvimento tecnológico no Brasil. Nesse sentido, conhecer o perfil desses estudantes possibilita um melhor planejamento das ações, tornando possível avançar nas políticas de inclusão, discutir, ampliar e melhorar a distribuição de recursos para a assistência.

Apesar do aumento quantitativo de estabelecimentos de ensino superior no Brasil na última década, pouco se conhece sobre a relação do ambiente acadêmico com a qualidade de vida da população universitária ${ }^{(5)}$. Diante das diversas áreas relacionadas à assistência estudantil, há uma carência de estudos sobre o perfil do estudante universitário, principalmente em relação a seu ingresso e inserção na vida universitária e sobre os aspectos que podem interferir na sua permanência ${ }^{(6)}$.

Ademais, a universidade apresenta-se como um ambiente propício para a efetivação de políticas promotoras de saúde, principalmente pelo fato dos indivíduos estarem em uma fase de transição para a vida adulta, em que a adoção de hábitos saudáveis repercutirá de forma positiva durante toda a fase do desenvolvimento humano. Considerando a universidade um ambiente que agrega aprendizagem e desenvolvimento e influencia a qualidade de vida de seus membros ${ }^{(7)}$, iniciativas promotoras de saúde no contexto universitário podem contribuir na capacitação dos indivíduos para torná-los autônomos em cuidar da própria saúde, oferecendo também ferramentas para o exercício da cidadania e o incentivo da participação social na formulação e manutenção 
das ações promotoras de saúde ${ }^{(7)}$. Considerando a universidade um ambiente que agrega aprendizagem e desenvolvimento e influencia a qualidade de vida de seus membros ${ }^{(7)}$. Para isso, são necessárias ações sob a ótica da promoção da saúde que possibilitem uma reflexão sobre a forma de viver e conviver da comunidade acadêmica, vislumbrando um ambiente saudável.

Pouco se conhece ainda sobre a relação do ambiente acadêmico com a saúde da população universitária. Sabe-se que as exigências da vida universitária demandam do estudante habilidades cognitivas e emocionais complexas para o manejo de elevadas expectativas em relação ao futuro, das pressões do mercado de trabalho e das frustrações que possam surgir nessa nova fase da vida. Essa situação predispõe o estudante a uma elevada prevalência de problemas psicoafetivos, os quais, em muitos casos, são identificados tardiamente e abordados de forma inadequada ${ }^{(8)}$.

Nesse sentido, o presente estudo tem como objetivo caracterizar o perfil sociodemográfico e clínico de estudantes universitários acolhidos em um serviço de saúde.

\section{MÉTODOS}

Trata-se de um estudo descritivo e retrospectivo, baseado na análise de prontuário de estudantes matriculados em uma instituição pública de ensino superior que utilizaram o serviço de saúde no período de 2011 a 2015. Esse período foi escolhido por abarcar estudantes desde o ingresso na universidade até a conclusão do curso.

Esses estudantes eram acolhidos pelo Núcleo de Apoio ao Estudante (NAE), um setor localizado dentro do campus universitário, que foi criado em 2010 para atender as demandas de assistência estudantil descritas no PNAES. Esse serviço estrutura suas ações por meio de uma equipe multidisciplinar, composta por médico, enfermeiro, psicólogo, assistente social e pedagogo. Além de atuar na lógica da Saúde Coletiva, possui o Hospital Universitário como retaguarda para encaminhamentos de média e alta complexidade, além de um ambulatório de especialistas para acolher os estudantes. O NAE funciona como um espaço de escuta e acolhimento, visando oferecer ao estudante uma possibilidade de compreensão/resolução das suas demandas de ordem socioeconômica, pedagógica, psicossocial ou de saúde. A demanda do serviço é espontânea e em todos os casos é realizado um acolhimento inicial e posterior, direcionamento para o profissional que possa atender às necessidades do estudante. O setor, além do acolhimento das demandas de saúde, coordena o programa de auxílio permanência do campus, que visa garantir a permanência dos estudantes de graduação que se encontram em estado de vulnerabilidade socioeconômica por meio da concessão de auxílios proveniente do PNAES.

O serviço funciona na Universidade Federal de São Paulo - campus Guarulhos, localizado numa região periférica e de elevada vulnerabilidade da grande São Paulo, atendendo aos requisitos do REUNI de promover a interiorização e descentralização das instituições de ensino superior. As características socioeconômicas da população universitária apontam para um elevado percentual de estudantes em vulnerabilidade socioeconômica, com renda mensal familiar de até dois salários mínimos $^{(9)}$, de forma que o recebimento do auxílio financeiro possibilita a permanência de aproximadamente $20 \%$ do total de estudantes matriculados nos cursos de graduação, acima da média nacional de aproximadamente $11 \%$ de estudantes atendidos por bolsas de permanência ${ }^{(2)}$. Esse fato repercute na condição de saúde do estudante, o que demanda um olhar ampliado sobre suas demandas de saúde.

Constavam nos prontuários analisados informações coletadas pelo profissional responsável pelo atendimento, as quais eram referentes à identificação pessoal do usuário (nome, endereço, telefone, sexo, gênero, idade, cidade de origem, local de moradia, curso e atividade profissional) e uma breve descrição da queixa inicial e do encaminhamento indicado. Foram incluídos no estudo prontuários de estudantes dos cursos de graduação, sendo excluídos prontuários de estudantes de pós-graduação.

Para traçar o perfil sociodemográfico e clínico dos estudantes, foram utilizadas as seguintes variáveis: sexo (masculino; feminino), gênero (cisgênero; transgênero), idade, curso (Ciências Sociais; Letras; Pedagogia; História; História daArte; Filosofia), ocupação, tipo de moradia (família; república estudantil; outros), existência de doença pré-existente e, por fim, o motivo da procura pelo serviço. Esses cursos foram escolhidos por serem os cursos de graduação do campus onde a pesquisa foi desenvolvida. A partir das informações obtidas dos prontuários, realizou-se a sistematização do material em planilha, seguida de uma análise descritiva com cálculo de prevalência. A análise foi realizada com o auxílio do software R.

O projeto de pesquisa foi submetido e aprovado ao Comitê de Ética em Pesquisa da Universidade Federal de São Paulo, sob o protocolo 1.388.092. Os dados foram coletados por meio da análise de prontuários, sendo dispensada a assinatura do Termo de Consentimento Livre e Esclarecido.

\section{RESULTADOS}

Foram elegíveis para o estudo 457 prontuários. As perdas foram de 40,3\% (n=309), totalizando 766 prontuários analisados. A maior parte da amostra era do sexo feminino, na faixa etária de 20 a 29 anos, residentes com seus familiares e cisgênero. (Tabela I) 
Tabela I - Descrição da amostra segundo sexo, gênero, curso, faixa etária e local de moradia (n=457). Guarulhos, São Paulo, 2016.

\begin{tabular}{|c|c|c|c|}
\hline Variáveis & & n & $\%$ \\
\hline \multirow[t]{2}{*}{ Sexo } & Feminino & 318 & 69,6 \\
\hline & Masculino & 139 & 30,4 \\
\hline \multirow[t]{2}{*}{ Gênero } & Cisgênero & 455 & 99,5 \\
\hline & Transgênero & 02 & 0,5 \\
\hline \multirow[t]{6}{*}{ Curso } & Ciências Sociais & 96 & 21 \\
\hline & Filosofia & 58 & 12,7 \\
\hline & História & 59 & 12,9 \\
\hline & História da Arte & 30 & 6,6 \\
\hline & Letras & 122 & 26,7 \\
\hline & Pedagogia & 92 & 20,1 \\
\hline \multirow[t]{6}{*}{ Idade (anos) } & $15-19$ & 12 & 2,6 \\
\hline & $20-29$ & 239 & 52,3 \\
\hline & $30-39$ & 125 & 27,4 \\
\hline & $40-49$ & 55 & 12 \\
\hline & $50-59$ & 23 & 05 \\
\hline & $60-69$ & 03 & 0,7 \\
\hline \multirow[t]{3}{*}{ Local de Moradia } & Família & 304 & 66,5 \\
\hline & República & 109 & 23,9 \\
\hline & Outro & 44 & 9,6 \\
\hline Total & & 457 & 100 \\
\hline
\end{tabular}

Cerca de metade dos estudantes não trabalham $(n=257)$, dedicando o seu tempo exclusivamente às atividades acadêmicas. Dentre os que trabalham $(n=200)$, a maioria atua como estagiário $(36,5 \%)$, seguido por profissionais da área de educação $(32,5 \%)$ e funções administrativas $(11,5 \%)$. (Tabela II)

Tabela II - Descrição das profissões dos estudantes trabalhadores (n=200). Guarulhos, São Paulo, 2016.

\begin{tabular}{lcc}
\hline Profissão & n & \% \\
\hline Aposentado & 01 & 0,5 \\
Atriz & 01 & 0,5 \\
Autônomo(a) & 14 & 7 \\
Comércio & 05 & 2,5 \\
Dona de casa & 01 & 0,5 \\
Educação & 65 & 32,5 \\
Empresário & 01 & 0,5 \\
Estagiário(a) & 73 & 36,5 \\
Funções administrativas & 23 & 11,5 \\
Informática & 02 & 1 \\
Marketing/Publicidade & 05 & 2,5 \\
Militar & 02 & 1 \\
Músico & 01 & 0,5 \\
Saúde & 04 & 2 \\
Servidor(a) Público(a) & 02 & 1 \\
\hline Total & 200 & 100 \\
\hline
\end{tabular}


No momento do acolhimento, os estudantes responderam se tinham alguma doença preexistente. A maioria (72,2\%) relatou não ter doenças preexistentes. Dentre os que responderam possuir alguma patologia, prevaleceram as doenças psicossociais (23\%), como depressão, ansiedade, síndrome do pânico, seguidas pelas doenças crônicas não transmissíveis (DCNT), como hipertensão e diabetes $(11 \%)$ e as doenças gastrointestinais (10,2\%), tais como: gastrite e síndrome do intestino irritável. (Tabela III)

Tabela III - Descrição das doenças preexistentes relatadas pelos estudantes (n=127). Guarulhos, São Paulo, 2016.

\begin{tabular}{lcc}
\hline Doença preexistente & $\mathbf{n}$ & $\mathbf{\%}$ \\
\hline Câncer & 02 & 1,6 \\
DCNT & 14 & 11 \\
Dermatológica & 08 & 6,3 \\
Ginecológica & 11 & 8,7 \\
Psicossocial & 29 & 22,8 \\
Cardiológica & 04 & 3,2 \\
Congênita & 09 & 7 \\
Tireóide & 06 & 4,7 \\
Gastrointestinais & 13 & 10,2 \\
Neurológica & 04 & 3,2 \\
Oftalmológica & 04 & 3,2 \\
Osteomuscular & 11 & 8,7 \\
Urológica & 03 & 2,4 \\
DST & 09 & 7 \\
\hline Total & 127 & 100 \\
\hline
\end{tabular}

Em relação ao que motivou o estudante a procurar o serviço de saúde, a maioria desejava encaminhamento para especialista médico $(n=225)$ no intuito de realizar consultas de rotina e/ou seguimento de tratamento de saúde; seguido de questões psicossociais $(n=68)$ e acolhimento e avaliação $(n=68)$. Foram incluídas na categoria "acolhimento e avaliação" as demandas de estudantes que buscavam o atendimento para uma escuta e acolhimento, sem demonstrarem uma queixa específica. (Tabela IV)

Tabela IV - Descrição da queixa inicial de saúde que motivou a procura pelo serviço (n=457). Guarulhos, São Paulo, 2016.

\begin{tabular}{lcc}
\hline Motivo do Atendimento & $\mathbf{n}$ & $\mathbf{\%}$ \\
\hline Acolhimento e avaliação & 68 & 14,9 \\
Cefaléia & 07 & 1,5 \\
Cessação do tabagismo & 02 & 0,5 \\
Dor/Lesão osteomuscular & 20 & 4,4 \\
Emagrecimento & 05 & 1,1 \\
Encaminhamento & 225 & 49,2 \\
Estresse/Fadiga & 03 & 0,6 \\
Gestante & 05 & 1,1 \\
HAS & 01 & 0,2 \\
Queixa gastrointestinal & 10 & 2,2 \\
Queixa ginecológica & 09 & 2 \\
Queixa odontológica & 02 & 0,5 \\
Queixa oftalmológica & 03 & 0,6 \\
Queixa respiratória & 01 & 0,2 \\
Queixa/Lesão dermatológica & 17 & 3,7 \\
Queixa urológica/sexual & 05 & 1,1 \\
Queixa vocal & 03 & 0,6 \\
Questões psicossocias & 68 & 14,9 \\
Uso/Abuso drogas & 01 & 0,2 \\
Violência sexual & 02 & 0,5 \\
\hline Total & 457 & 100 \\
\hline
\end{tabular}




\section{DISCUSSÃO}

A maior parte dos estudantes do presente estudo que utilizaram o serviço de saúde era do sexo feminino. A predominância de mulheres pode ter ocorrido pelo fato dos cursos de Letras e Pedagogia, além de concentrarem o maior número de vagas, serem majoritariamente cursos escolhidos pelo sexo feminino. Outros estudos com estudantes universitários também apontaram para a predominância feminina na procura por atendimento à saúde ${ }^{(2,10)}$. Uma questão importante são os fatores socioculturais brasileiros, que fazem com que as mulheres tenham tendência a terem mais cuidado com a saúde e adotarem hábitos preventivos, como realizar consultas médicas regulares.

Apesar do reduzido quantitativo de estudantes autodeclarados transgêneros, o fato de tê-los na universidade suscita o debate sobre a inclusão e o respeito à diversidade de gênero. Recentemente, diversas universidades estão reconhecendo o direito básico da adoção do nome social por membros da comunidade acadêmica, o que coaduna com a política de ações afirmativas recém-implementadas no ensino superior.

O trabalho também é importante componente na análise da saúde do estudante. A classificação de diferentes formas de interação do estudante com o trabalho definiu: o estudante em tempo integral, aquele que é mantido pela família e dedica-se exclusivamente aos estudos; o estudante/trabalhador, que trabalha mas ainda é dependente financeiramente da família; e o trabalhador/estudante, que, além de não depender da família, ainda contribui com o orçamento doméstico ${ }^{(11)}$. A sobrecarga de afazeres diários impacta na saúde, o que desencadeia uma série de sintomas relacionados ao estresse.

Um estudo que avaliou o reflexo das políticas de inclusão adotadas pelo governo em todos os cursos de graduação evidenciou que há um número cada vez menor de estudantes ricos ingressantes na educação superior, sobretudo nos cursos de alta demanda, como Medicina. A renda familiar média de estudantes de cursos como História e Pedagogia é de até 03 salários mínimos, o que explica o elevado percentual de estudantes que precisam trabalhar para se manter na universidade ou ajudar nas despesas da família ${ }^{(12)}$. Nesse sentido, as políticas de assistência estudantil proporcionam a permanência do estudante de baixa renda no ensino superior.

É importante observar que uma parcela dos estudantes do presente estudo adentra a universidade já com problemas de saúde, principalmente questões psicossociais, doenças crônicas não transmissíveis e doenças gastrointestinais. A fase de ingresso na universidade coincide com períodos de questionamentos acerca de valores, crenças, autonomia, relações familiares e com a sociedade. Diante disso, é possível que estudantes adotem um comportamento de risco à saúde, tais como: consumo de álcool, tentativa de suicídio, excesso de peso e hábitos alimentares poucos saudáveis, o que pode agravar patologias preexistentes ${ }^{(13)}$. Além disso, essas patologias têm estreita relação com o estresse ${ }^{(14)}$, a inatividade física ${ }^{(15)}$ e o sofrimento psíquico ${ }^{(8)}$ vivenciados no ingresso na universidade.

Quanto às queixas que motivaram a procura pelo serviço de saúde na atual pesquisa, os estudantes majoritariamente solicitaram encaminhamento para especialista médico, o que se deve ao fato do sistema de saúde pública demorar um longo período de espera para encaminhamento para especialistas e dos estudantes verem no hospital universitário uma oportunidade de atendimento mais rápido e de qualidade.

As queixas psicossociais e necessidade de acolhimento e avaliação também foram as mais prevalentes no atual estudo. Quanto às questões psicossociais, é relevante observar que os estudantes apresentam demandas de saúde que, uma vez não acolhidas, podem evoluir para questões mais complexas e impactar na sua qualidade de vida e permanência na universidade. Um estudo do perfil de atendimento de um serviço de psicologia voltado para estudantes universitários evidenciou que as queixas iniciais estavam relacionadas com situações vivenciadas na universidade, tais como: ansiedade frente às avaliações; dificuldade para se distanciar da família e estabelecer novos vínculos; problemas relacionados ao abuso de álcool e outras drogas; e depressão ${ }^{(10)}$.

Uma análise de indicadores de vulnerabilidade e bem-estar psicológico em estudantes universitários demonstrou que os sinais indicadores de estresse, burnout, ansiedade e depressão na população estudada revelaram-se comuns, sendo mais prevalente no sexo feminino. Sugerem-se algumas ações que podem minimizar as dificuldades no processo adaptativo à universidade, tais como: incentivo à convivência com a família, ampliação do número de profissionais de assistência psicossocial e políticas voltadas à saúde da mulher ${ }^{(8)}$. O sofrimento psíquico permeia toda a vida acadêmica, sendo mais elevado no momento de ingresso e término do curso. Nessas situações, o estudante se depara com um contexto desconhecido e repleto de incertezas, que se intensificam no momento de inserção no mercado de trabalho ${ }^{(16)}$. Diante disso, o serviço de saúde para os estudantes configura-se como um espaço privilegiado de escuta das demandas dos discentes, o que favorece o vínculo com a instituição e atendimento às necessidades de forma personalizada e individualizada.

O cuidado assume um papel de acolher a demanda do outro, no sentido de estar com o outro e possibilitar o exercício da liberdade de escolha e responsabilização pelo tratamento ${ }^{(17)}$. Práticas em saúde na perspectiva da promoção da saúde partem do pressuposto de que o conhecimento é construído através da parceria entre usuários e profissionais e de que a busca da saúde é centrada no ser humano saudável, tanto individual como coletivamente. Empoderá-lo permite uma maior interação com o seu estado de saúde, uma maior consciência para tomada de decisão e autonomia para fazer escolhas ${ }^{(18)}$.

Os resultados demonstram que os estudantes carecem de um serviço de atendimento multidisciplinar em saúde, onde suas demandas possam ser acolhidas e encaminhadas, quando necessário. É importante salientar que as queixas dos estudantes estão 
direta ou indiretamente relacionadas à situação de estresse vivenciada em grandes centros urbanos, como longas distâncias percorridas em transporte público, a sobrecarga de afazeres e as dificuldades socioeconômicas. Essas questões impactam diretamente a saúde, surgindo ou agravando patologias relatadas pelos estudantes.

Esse fato demanda ações voltadas para a promoção da saúde, como uma política de alimentação saudável no campus, ações que incentivem a atividade física regular e atividades culturais de lazer e relaxamento. Cabe à equipe de saúde, além de acolher as demandas, perceber questões ambientais da universidade e do entorno que podem impactar negativamente na saúde dos estudantes. Diante dos resultados encontrados, carece aprofundar a pesquisa no desmembramento das queixas psicossociais e trabalhar mais especificamente com o estudante trabalhador, principalmente do turno noturno, fazendo um recorte de renda e de condições de vida.

O estudo elucidou importantes características desse "novo" estudante universitário, trazendo elementos relacionados a sua saúde que podem contribuir na elaboração e na avaliação das políticas de assistência estudantil vigentes.

O presente estudo teve como limitação o fato da população de estudantes ser de um único campus e de cursos com características similares, o que traz uma homogeneidade no perfil estudantil. Outra limitação foi a significativa perda de informações devido ao quantitativo de prontuários preenchidos de forma incompleta, reduzindo o tamanho da amostra.

\section{CONCLUSÃO}

O estudo evidenciou o perfil dos estudantes atendidos no serviço de saúde, o qual era predominantemente composto por mulheres, na faixa etária de 20 a 29 anos, residentes com suas famílias e atuantes no mercado de trabalho. Apesar de a maioria não referir doenças preexistentes, quando presentes estavam relacionadas à questões psicossociais, doenças crônicas e gastrointestinais. Os atendimentos em saúde eram motivados por necessidades de encaminhamento para especialista, acolhimento e avaliação, e queixas psicossociais.

\section{REFERÊNCIAS}

1. Britto LPL, Silva EO, Castilho KC, Abreu TM. Conhecimento e formação nas IES periféricas perfil do aluno "novo" da educação superior. Avaliação [Internet]. 2008 [acesso em 2016 Dez 10]; 13(3):777-91. Disponível em: http://www.scielo. br/scielo.php?script=sci_arttext\&pid=S1414-40772008000300008\&lng=pt\&nrm=iso\&tlng=pt

2. Fórum Nacional de Pró-Reitores de Assuntos Comunitários e Estudantis - Fonaprace. Perfil socioeconômico e cultural dos estudantes de graduação das universidades federais brasileiras. Brasília: Associação Nacional dos Dirigentes das Instituições Nacionais de Ensino Superior; 2011.

3. Brasil. Programa Nacional de Assistência Estudantil - Lei 7.234 de 24 de Julho de 2010 [acesso em 2016 Dez 10]. Disponível em: http://www.ufjf.br/proae/files/2008/10/DECRETO-N\%C2\%BA-7234.pdf

4. Westphal MF. O movimento de cidades/municípios saudáveis: um compromisso com a qualidade de vida. Ciênc Saúde Coletiva [Internet]. 2000 [acesso em 2016 Ago 15]; 5(1):39-51. Disponível em: http://www.scielo.br/pdf/csc/v5n1/7078. pdf

5. Ribeiro IM, Patrício ZM, Reis AE, Santos EM. Repercussões do processo ensino-aprendizagem na qualidade de vidasaúde de acadêmicos: entre possibilidades e limitações. REME Rev Min Enferm [Internet]. 2010 [acesso em 2016 Dez 10]; 14(1):96-102. Disponível em: http://www.reme.org.br/artigo/detalhes/93

6. Heringer R, Honorato GS. Elementos para uma análise dos estudantes cotistas e bolsistas no curso de pedagogia da UFRJ. Caderno CRH [Internet]. 2015 [acesso em 2016 Set 29];28(74):341-8. Disponível em: http://doi.org/10.1590/S010349792015000200007

7. Mello ALSF, Moyses ST, Moyses SJ. A universidade promotora de saúde e as mudanças na formação profissional. Interface Comun Saúde Educ [Internet]. 2010 [acesso em 2016 Out 01];14(34):683-92. Disponível em: http://www.scielo.br/pdf/ icse/v14n34/aop0210.pdf

8. Padovani RC, Neufeld CB, Maltoni JB, Leopoldo NF, Souza WF, Cavalcanti HAF, et al. Vulnerabilidade e bem-estar psicológicos do estudante universitário. Rev Bras Ter Cogn [Internet]. 2014 [acesso em 2016 Dez 10];10(1):2-10. Disponível em: https://dx.doi.org/10.5935/1808-5687.20140002

9. Cruz FM, Cespedes JG. O perfil socioeconômico e cultural dos estudantes da Universidade Federal de São Paulo. São Paulo: UFSP; 2012.

10. Peres RS, Santos MA, Coelho HMB. Perfil da clientela de um programa de pronto-atendimento psicológico a estudantes universitários. Psicol Estud [Internet]. 2004 [acesso 2015 Nov 01];9(1):47-54. Disponível em: http://www.scielo.br/pdf/pe/ v9n1/v9n1a07.pdf. 
11. Moraes CAS, Botelho TM, Fonseca TA, Almeida DO, Bastos JC. O estudante do ensino superior: identificando categorias de análise. Vértices [Internet]. 2011 [acesso em 2016 Nov 01];13(03):205-18. Disponível em: http://dx.doi. org/10.19180/1809-2667.20110034

12. Ristoff D. O novo perfil do campus brasileiro: uma análise do perfil socioeconômico do estudante de graduação. Avaliação [Internet]. 2014 [acesso em 2016 Nov 10]; 19(3):723-47. Disponível em: http://www.scielo.br/scielo.php?script=sci_ arttext\&pid=S1414-40772014000300010\&lng=pt\&nrm=iso\&tlng=en

13. Faria YO, Gandolfi L, Moura LBA. Prevalência de comportamentos de risco em adulto jovem e universitário. Acta Paul Enferm [Internet]. 2014 [acesso em 2016 Nov 10];27(6):591-5. Disponível em: http:/www.scielo.br/pdf/ape/v27n6/19820194-ape-027-006-0591.pdf

14. Feldman L, Goncalves L, Chacón-Puignau G, Zaragoza J, Bagés N, Pablo J. Relaciones entre estrés académico, apoyo social, salud mental y rendimiento académico em estudiantes universitarios venezolanos. Universitas Psychologica [Internet]. 2008 [acesso em 2016 Set 29];7(3):739-51. Disponível em: http://www.redalyc.org/articulo.oa?id=64770311

15. Sousa TF, José HPM, Barbosa AR. Condutas negativas à saúde em estudantes universitários brasileiros. Ciênc Saúde Coletiva [Internet]. 2013 [acesso em 2017 Jan 02];18(12):3563-75. Disponível em: http://dx.doi.org/10.1590/S141381232013001200013

16. Carvalho EA, Bertolini SMMG, Milani RG, Martins MC. Índice de ansiedade em universitários ingressantes e concluintes de uma instituição de ensino superior. Ciênc Cuid Saúde [Internet]. 2015 [acesso em 2016 Nov 01];14(3):1290-8. Disponível em: periodicos.uem.br/ojs/index.php/CiencCuidSaude/article/view/23594

17. Anéas TV, Ayres JRCM. Significados e sentidos das práticas de saúde: a ontologia fundamental e a reconstrução do cuidado em saúde. Interface Comun Saúde Educ [Internet]. 2011 [acesso em 2015 Dez 10];15(38):651-62. Disponível em: http:// www.scielo.br/scielo.php?script=sci_arttext\&pid=S1414-32832011000300003\&lng=en

18. Salci MA, Maceno P, Rozza SG, Silva DMGVS, Boehs AE, Heidemann ITSB. Educação em saúde e suas perspectivas teóricas: algumas reflexões. Texto \& Contexto Enferm [Internet]. 2013 [acesso em 2015 Ago 10];22(1):224-30. Disponível em: www.scielo.br/pdf/tce/v22n1/pt_27.pdf

\section{Endereço para correspondência:}

Lucíola D’Emery Siqueira

Universidade Federal de São Paulo - UNIFESP

Estrada do caminho velho, 333

Bairro: Jd. Nova Cidade

CEP: 07252-312 - Guarulhos - São Paulo - Brasil

E-mail: luciola.demery@gmail.com 